\title{
The Mitogen-Activated Protein Kinase Gene MAF1 Is Essential for the Early Differentiation Phase of Appressorium Formation in Colletotrichum lagenarium
}

\author{
Kaihei Kojima, Taisei Kikuchi, Yoshitaka Takano, Eriko Oshiro, and Tetsuro Okuno \\ Laboratory of Plant Pathology, Graduate School of Agriculture, Kyoto University, Kyoto 606-8502, Japan \\ Submitted 11 March 2002. Accepted 4 September 2002.
}

Colletotrichum lagenarium, the causal agent of cucumber anthracnose, invades host plants by forming a specialized infection structure called an appressorium. In this fungus, the mitogen-activated protein kinase (MAPK) gene CMK1 is involved in several steps of the infection process, including appressorium formation. In this study, the goal was to investigate roles of other MAPKs in $C$. lagenarium. The MAPK gene MAF1, related to Saccharomyces cerevisiae MPK1 and Magnaporthe grisea MPS1, was isolated and functionally characterized. The mafl gene replacement mutants grew normally, but there was a significant reduction in conidiation and fungal pathogenicity. The $M$. grisea mps 1 mutant forms appressoria, but conidia of the C. lagenarium maf1 mutants produced elongated germ tubes without appressoria on both host plant and glass, on which the wild type forms appressoria, suggesting that $M A F 1$ has an essential role in appressorium formation on inductive surfaces. On a nutrient agar, wild-type conidia produced elongated germ tubes without appressoria. The morphological phenotype of the wild type on the nutrient agar was similar to that of the maf1 mutants on inductive surfaces, suggesting repression of the $M A F 1$-mediated appressorium differentiation on the nutrient agar. The $c m k 1$ mutants failed to form normal appressoria but produced swollen, appressorium-like structures on inductive surfaces, which is morphologically different from the mafl mutants. These findings suggest that $M A F 1$ is required for the early differentiation phase of appressorium formation, whereas $C M K 1$ is involved in the maturation of appressoria.

Anthracnose fungi belonging to the genus Colletotrichum cause severe diseases on a wide range of crops and ornamental plants (Agrios, 1988). C. lagenarium (Pass.) Ellis \& Halst. (syn. C. orbiculare (Berk. \& Mont.) Arx) is the causal agent of cucumber anthracnose. This fungus forms a swollen infection structure called an appressorium. In several cases, it is known that fungal pathogens form appressoria in response to distinct physical and chemical cues in their environment. The bean rust fungus, Uromyces appediculatus, forms appressoria upon en-

Corresponding author: Y. Takano; E-mail: ytakano@kais.kyoto-u.ac.jp

Current address of T. Kikuchi: Forestry and Forest Products Research Institute, Tsukuba, Ibaraki 305-8687, Japan.

Current address of E. Oshiro: Kizakura Sake Brewing Co., Ltd., Fushimiku, Kyoto 612-8046, Japan.

Nucleotide and amino acid sequence data for $M A F 1$ are to be found in the GenBank database under accession number AY064246. countering ridges formed by the guard cells surrounding a stomatal opening. Hoch and colleagues (1987) demonstrated that artificial ridges mimicking those formed by guard cells are sufficient to induce appressorium formation in this fungus. On the other hand, the rice blast fungus, Magnaporthe grisea, forms appressoria efficiently on inert hydrophobic surfaces in a droplet of water, whereas appressoria are much less efficiently formed on hydrophilic surfaces, suggesting that surface hydrophobicity induces appressorium formation (Lee and Dean, 1994). In $M$. grisea, the cAMP signaling pathway has been shown to be involved in appressorium differentiation through sensing of surface hydrophobicity (Adachi and Hamer, 1998; Choi and Dean, 1997; Lee and Dean, 1993). In C. lagenarium, knockout analysis of $R P K 1$, encoding a regulatory subunit of cAMP-dependent protein kinase (PKA), indicated that proper regulation of PKA is required for appressorium function (Takano et al., 2001a).

Mitogen-activated protein kinase (MAPK) cascades have also been shown to play pivotal roles in fungal infection processes (Xu, 2000). MAPK cascades generally consist of three conserved kinases, MAP kinase kinase kinase (MAPKKK), MAP kinase kinase (MAPKK), and MAPK, which function sequentially and mediate appropriate cellular responses to distinct environmental inputs (Nishida and Gotoh, 1993; Schaeffer and Webber, 1999). The budding yeast Saccharomyces cerevisiae has five MAPKs, encoded by FUS3, KSS1, HOG1, MPK1 (SLT2), and SMK1, respectively (Herskowitz, 1995; Hunter and Plowman, 1997). These MAPK cascades regulate fungal growth and differentiation processes such as pheromone response, pseudohyphal growth, osmoregulation, cell wall integrity, and ascospore formation. Fus3/Kss1-related MAPK genes of several phytopathogenic fungi, including $P M K 1$ in $M$. grisea, CHK1 in Cochliobolus heterostrophus, UBC3 (KPP2) in Ustilago maydis, CMK1 in C. lagenarium, BMP1 in Botrytis cinerea, FMK1 in Fusarium oxysporum, and PTK1 in Pyrenophora teres, play important roles in fungal pathogenicity (Di Pietro et al., 2001; Lev et al., 1999; Mayorga and Gold, 1999; Muller et al., 1999; Ruiz-Roldan et al., 2001; Takano et al., 2000; Xu and Hamer, 1996; Zheng et al., 2000). Furthermore, $P M K 1, C H K 1, C M K 1, F M K 1$, and PTK1 are required for appressorium formation, suggesting conserved roles of Fus3/Kss1-related MAPKs in the formation of infection structures by plant pathogens. However, knockout mutants of the MAPKs in each of the fungi show different phenotypes in growth, conidiation, and germination, which suggests diverse roles of Fus3/Kss1-related MAPKs in phytopathogenic fungi.

In contrast with the extensive investigation of Fus3/Kss1-related MAPKs, the roles of Mpk1-related MAPK pathways are less well studied. At present, functional analysis of the MPK1- 
type MAPK gene is limited to MPS1 in M. grisea (Xu et al., 1998). It is known that the Mpk1 pathway in $S$. cerevisiae is a part of the signaling pathway controlling cell wall integrity (Lee et al., 1993). M. grisea mps 1 mutants are hypersensitive to cell-wall-digesting enzymes, suggesting that MPS1 is also involved in cell wall integrity (Xu et al., 1998). Phenotypic analysis of mps 1 mutants during infection indicated that MPS1 is dispensable for appressorium formation but is required for penetration by appressoria (Xu et al., 1998).

In this report, we identify the $C$. lagenarium MAPK gene, $M A F 1$ (MAPK essential for appressorium formation), related to MPK1 of S. cerevisiae and MPS1 of M. grisea. We have investigated the roles of the $M A F 1$-encoded MAPK cascade in $C$. lagenarium by assessing the phenotype of knockout mutants. The mafl mutants grew normally but exhibited significant reductions in conidiation and pathogenicity. In contrast with $M$. grisea mps1 mutants, which are able to form appressoria, $C$. lagenarium mafl mutants failed to form appressoria on both host plant and glass, on which the wild type forms appressoria, demonstrating that the Maf1 MAPK is essential for appressorium formation in $C$. lagenarium. Further analyses, including comparison of mafl mutants with $\mathrm{cmkl}$ mutants having a defect in appressorium formation, indicated that two MAPKs (Maf1 and Cmk1) regulate distinct steps in appressorium development in $C$. lagenarium.

\section{RESULTS}

Identification of the MAF1 gene encoding

a MAPK related to yeast Mpk1.

We isolated MPK1-related MAPK genes in C. lagenarium by polymerase chain reaction (PCR) using degenerate oligonucleotide primers designed from amino acid sequences con- served in Mpk1-type MAPKs in other organisms. A cDNA pool synthesized from mRNA isolated from conidia during appressorium formation (after $2 \mathrm{~h}$ of incubation) was used as a template for PCR screening (Inagaki et al., 2000). Amplified products were cloned into pBluescript and sequenced. The DNA sequence of an insert fragment in one PCR clone, pMP1, showed a high degree of similarity to Mpk1-type MAPKs in other organisms. The insert DNA was used as a probe to isolate genomic clones containing the MPK1-related MAPK gene of $C$. lagenarium, designated $M A F 1$. The entire nucleotide sequence of the $M A F 1$ gene was determined. MAFl contains an open reading frame composed of 418 codons and four introns (Fig. 1 and data not shown). Maf1 contains the sequence TEY, a site for threonine and tyrosine phosphorylation required for kinase activation (Fig. 1). The deduced amino acid sequence of Maf1 was found to have $91 \%$ identity with $M$. grisea Mps1 and $60 \%$ with S. cerevisiae Mpk1.

\section{Gene disruption of MAF1.}

To investigate the roles of $M A F 1$ in $C$. lagenarium, a gene disruption strategy was employed. A gene replacement vector, pGDMAF1, containing a hygromycin B phosphotransferase (hph) gene cassette and 5' and 3' flanking regions of MAFl, was constructed and introduced into the wild-type strain 104-T (Fig. 2A). Eight of 20 hygromycin-resistant transformants formed colonies different from those of the wild type on nutrient agar plates (Fig. 2C). We tested whether MAF1 was disrupted in the transformants that exhibited the different colony phenotype. Gene replacement of $M A F 1$ was investigated by DNA gel blot analysis (Fig. 2B). Genomic DNAs were isolated from the wild-type 104-T; the transformant DMA8, which forms wild-type colonies; and the transformants DMA5, DMA13, DMA15, and DMA16, which form colonies distinct

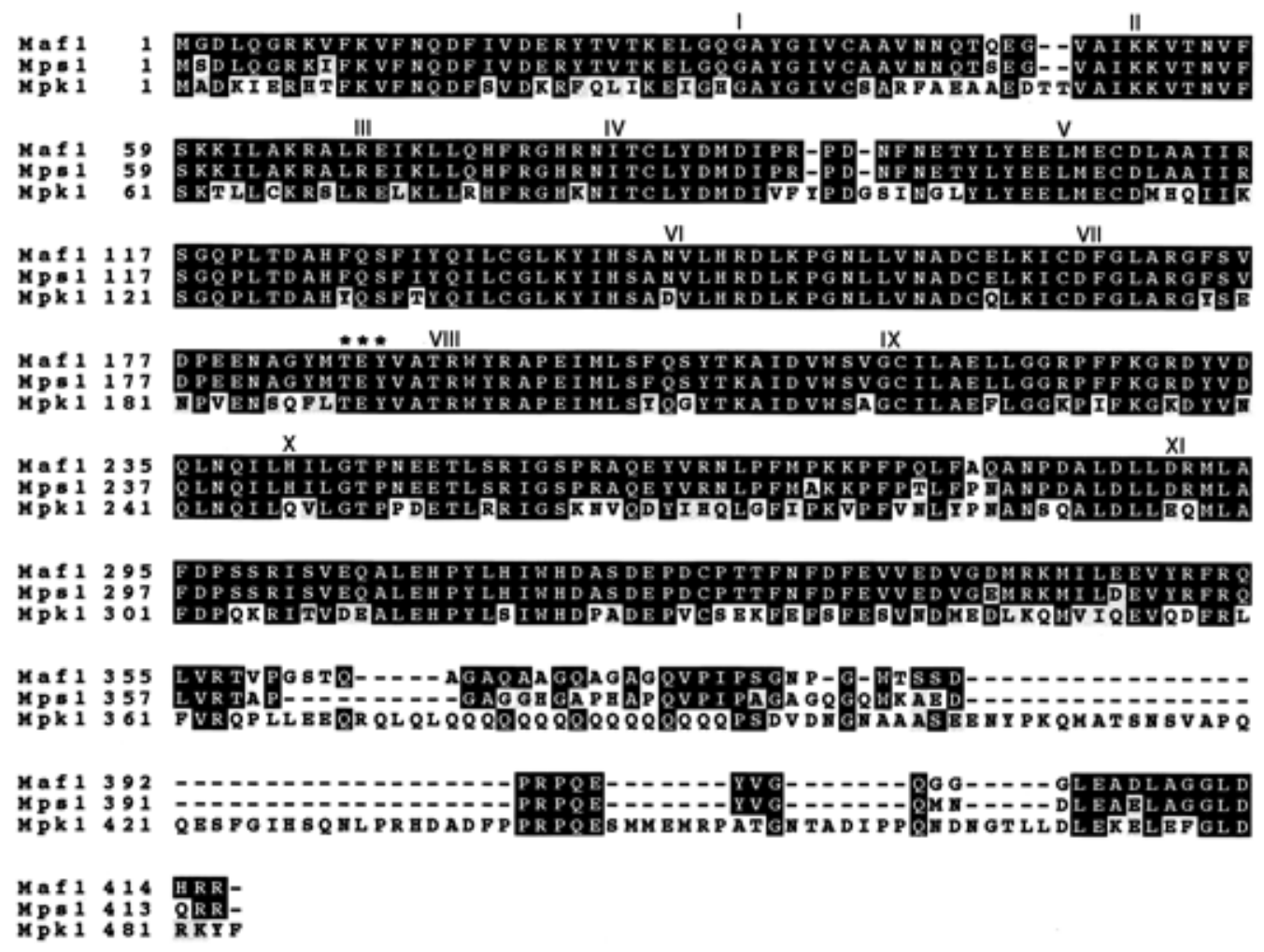

Fig. 1. The Colletotrichum lagenarium MAPK gene MAF1 related to Saccharomyces cerevisiae MPK1. Amino acid sequence alignment of Maf1 of $C$. lagenarium with Mpk1 of S. cerevisiae and Mps1 of Magnaporthe grisea. Sequences were aligned with the CLUSTAL W program (Thompson et al., 1994). Identical amino acids are indicated by white letters on a black background. Similar residues are indicated by a gray background. Gaps introduced for alignment are indicated by hyphens. The protein kinase domains are indicated by Roman numerals above sequences. Sequence TEY, which is required for kinase activation, is marked by asterisks. 
from those of the wild type. Isolated genomic DNAs were digested with Pst I and probed with a 6.7-kb Pst I fragment containing $M A F 1$. Strains 104-T and DMA 8 contained the common 6.7-kb Pst I fragment (Fig. 2B, lanes 1 and 6). DMA8 also contained additional bands, indicating ectopic integration of pGDMAF1. On the other hand, DMA5, DMA13, DMA15, and DMA16 did not contain the 6.7-kb Pst I fragment but contained common 2.8-kb and 3.8-kb PstI fragments (Fig. 2B, lanes 2 to 5 ), which is consistent with the lengths expected in gene replacement. We concluded that the MAF1 gene was disrupted in transformants DMA5, DMA13, DMA15, and DMA16. These mafl $\Delta$ strains grew normally, at growth rates slightly higher than that of the wild type on potato-dextrose agar (PDA) (Table 1 and Fig. 2C). Also, on minimal medium agar plates, the mafl mutants grew like the wild type (data not shown). These results indicate that $M A F 1$ is not essential for mycelial growth on nutrient agar plates. On the other hand, conidiation of the mafl mutants was significantly reduced, in comparison with that of the wild-type strain (Table 1 and Fig. 2C). The number of co-

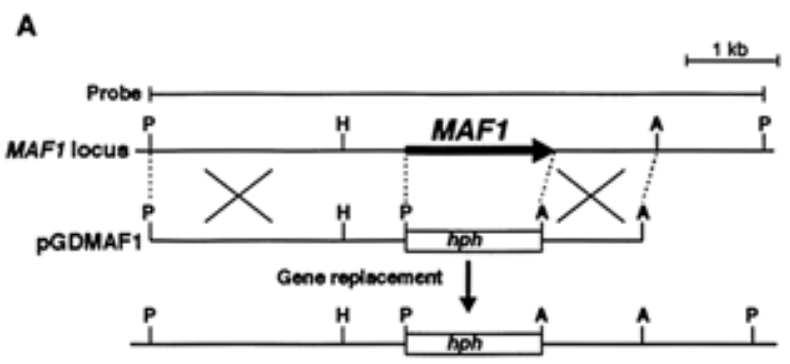

B

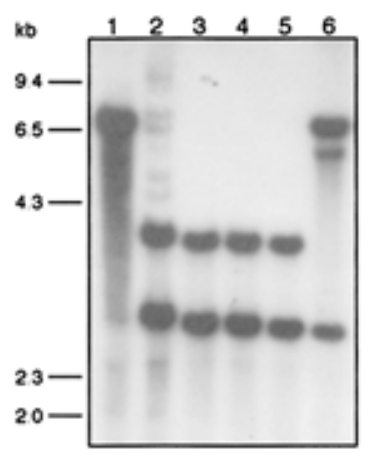

C

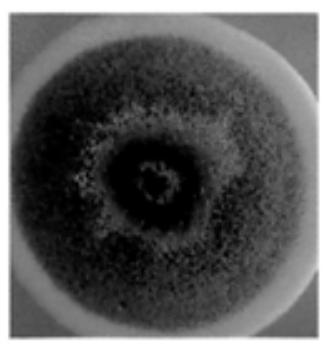

104- $\mathrm{T}$

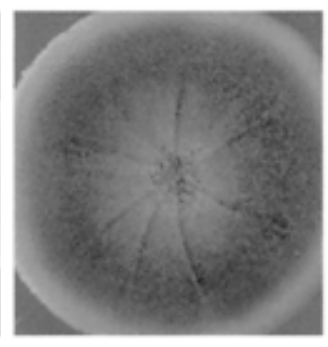

DMA5
Fig. 2. Gene disruption of $M A F 1$. A, $M A F 1$ locus and gene replacement vector pGDMAF1, containing a hygromycin-resistant gene $(h p h)$ cassette flanked by border sequences of $M A F 1$. By homologous recombination through double crossing, the entire region of $M A F 1$ was replaced by the $h p h$ cassette. B, Genomic DNA gel blot analysis of MAF1 gene replacement transformants. Genomic DNAs were extracted from the wild-type strain 104-T (lane 1); MAF1 gene replacement transformants DMA5, DMA13, DMA15, and DMA16 (lanes 2-5); and ectopic transformant DMA8 (lane 6). All genomic DNA samples were digested by Pst I. The blot was probed with the 6.7-kb PstI fragment shown in A. C, Colony morphology of the wild-type strain 104-T and maf1 $\triangle$ strain DMA5 incubated on potato-dextrose agar plates at $24^{\circ} \mathrm{C}$ for 14 days. nidia produced by the mafl mutants was approximately $10 \%$ of that produced by the wild type. To determine whether conidiation in the mafl mutants could be complemented by the introduction of $M A F 1$, the mafl mutant DMA5 was transformed with plasmid pCBMAF1, containing $M A F 1$ with the bialaphos resistance gene (Fig. 3A). Bialaphos-resistant transformants were obtained and analyzed by genomic DNA gel blot analysis (Fig. 3B). The analysis detected the 2.4-kb HindIII-PstI fragment containing $M A F 1$ in the transformants RMA1 and RMA2 but not in the mafl mutant DMA5, indicating that the MAF1 gene had been introduced into RMA1 and RMA2. In RMA2, one additional band was detected, suggesting integration of the truncated MAF1 fragment. In both RMA1 and RMA2, conidiation was similar to that of the wild-type strain (Table 1). We concluded that the MAFl-encoded MAPK pathway is involved in the conidiation of $C$. lagenarium.

As was mentioned earlier, it has been reported that $M P K 1$-type MAPKs are involved in cell wall integrity in several fungi, including the plant pathogen $M$. grisea. To evaluate whether MAF1 is involved in cell wall integrity, we investigated sensitivity to cell-wall-digesting enzymes in the mafl mutants. Mycelia of tested strains were treated with the cell-wall-digesting enzymes Driselase and $\beta$-D-glucanase, protoplasts released from the mycelia were examined 3 and $6 \mathrm{~h}$ after the treatment (Fig. 4). The number of protoplasts released by the mafl mutant was similar to that of the wild-type strain, which suggests that MAF1 is not essential for cell wall integrity in C. lagenarium.

\section{$M A F 1$ is essential for appressorium formation.}

Infection-related morphogenesis of the mafl $\Delta$ strains on a glass surface was investigated by microscopic observation. Germination of the mafl mutants on glass was the same as that of the wild type (Table 1). Conidia of the wild-type strain formed melanized appressoria on glass after $12 \mathrm{~h}$ of incubation, while germ tubes of the mafl mutants elongated continuously and failed to differentiate into appressoria (Fig. 5A and B and Table 1). The mafl mutants did not form appressoria, even after $48 \mathrm{~h}$ of incubation. The mafl mutants also failed to form appressoria on the surface of host cucumber cotyledons (Fig. $5 \mathrm{D}$ and $\mathrm{E})$. In contrast, RMA1 and RMA2, the $M A F 1$-introduced transformants of the mafl $1 \Delta$ strain DMA5, formed darkly melanized appressoria on both glass and host surfaces (Fig. 5C

Table 1. Characteristics of maf1 mutants of Colletotrichum lagenarium

\begin{tabular}{|c|c|c|c|c|}
\hline Strain $^{a}$ & $\begin{array}{l}\text { Vegetative } \\
\text { growth }^{b} \\
(\mathrm{~mm})\end{array}$ & $\begin{array}{l}\text { Conidiaa }^{c} \\
\left(\times \mathbf{1 0}^{6}\right)\end{array}$ & $\begin{array}{c}\text { Germination }^{\mathrm{d}} \\
(\%)\end{array}$ & $\begin{array}{c}\text { Appressorium } \\
\text { formation }^{\mathrm{e}} \\
(\%)\end{array}$ \\
\hline 104-T & $34.0 \pm 2.0$ & $54.3 \pm 22.5$ & $95.9 \pm 2.0$ & $95.8 \pm 2.1$ \\
\hline DMA5 & $39.3 \pm 1.1$ & $5.4 \pm 3.1$ & $98.3 \pm 1.2$ & $0.0 \pm 0.0$ \\
\hline DMA13 & $36.7 \pm 2.1$ & $5.4 \pm 1.8$ & $96.8 \pm 2.0$ & $0.0 \pm 0.0$ \\
\hline DMA15 & $38.3 \pm 2.1$ & $4.0 \pm 1.3$ & $92.8 \pm 6.1$ & $0.0 \pm 0.0$ \\
\hline DMA16 & $36.7 \pm 3.1$ & $3.7 \pm 0.5$ & $94.6 \pm 2.3$ & $0.0 \pm 0.0$ \\
\hline DMA8 & $33.3 \pm 2.3$ & $43.7 \pm 5.7$ & $94.8 \pm 0.9$ & $93.4 \pm 1.4$ \\
\hline RMA1 & $33.3 \pm 1.5$ & $53.3 \pm 9.1$ & $96.2 \pm 0.1$ & $94.4 \pm 1.6$ \\
\hline RMA2 & $34.0 \pm 1.0$ & $35.7 \pm 4.1$ & $93.1 \pm 0.2$ & $92.3 \pm 1.0$ \\
\hline
\end{tabular}

$\bar{a}$ Strains tested were the wild-type strain 104-T; MAF1-disrupted transformants DMA5, DMA13, DMA15, and DMA16; ectopic integration transformant DMA8; and two MAF1-reintroduced transformants of DMA5, designated RMA1 and RMA2.

${ }^{\mathrm{b}}$ Diameter of colonies grown on potato-dextrose agar at $24^{\circ} \mathrm{C}$ for 7 days. ${ }^{\mathrm{c}}$ Conidia collected from 7-day-old cultures.

${ }^{\mathrm{d}}$ Percentage of conidia that germinated after incubation on glass slides at $24^{\circ} \mathrm{C}$ for $12 \mathrm{~h}$. At least 200 conidia were examined to calculate percentage germination. The mean and standard deviation were calculated from three independent experiments.

${ }^{\mathrm{e}}$ Percentage of germinating conidia that formed appressoria after incubation for $12 \mathrm{~h}$. The mean and standard deviation were calculated from three independent experiments. 
and $\mathrm{F}$ and Table 1). From these results, we conclude that $M A F 1$ is essential for appressorium formation in C. lagenarium.

We compared the behavior of an mafl mutant with that of the wild type during conidial incubation on a glass surface with nuclei stained with 4',6-diamidino-2-phenylindole dihydrochloride (DAPI). After $3 \mathrm{~h}$ of incubation, conidia of both the mafl mutant DMA5 and the wild-type strain 104-T had germinated (more than 90\% germination; $n=300$ ), but nuclear division was not observed. Germinating conidia of the wild type formed appressoria accompanied by a single round of nuclear division, and one of the two daughter nuclei had moved to the appressorium by $6 \mathrm{~h}$ (Takano et al., 2001b, and this study). At $12 \mathrm{~h}$, the wild-type strain had formed highly melanized appressoria (more than $95 \%$ of germinated conidia formed appressoria; $n=300$ ) and had not undergone further nuclear division. Multiple nuclear divisions after the first nuclear division occurred in DMA5, in contrast to the wild-type strain. After $12 \mathrm{~h}$ of incubation, $60 \%$ of germinating conidia of DMA5 ( $n=200)$ contained four nuclei (Fig. 5G and H). At further long incubation, DMA5 continued nuclear division. These results suggest that the disruption of $M A F 1$ affected the nuclear duplication process during infection-related morphogenesis.

\section{$M A F 1$ is required for fungal pathogenicity.}

To investigate the pathogenicity of the mafl $1 \Delta$ strains, an infection assay was performed. Suspensions of conidia of strains 104-T, DMA5, and RMA1 were sprayed on detached cucumber leaves. Many yellowish lesions formed on cucumber leaves treated with the wild-type 104-T or with RMA1, while only a few lesions formed on leaves treated with the mafl $\Delta$ strain DMA5 (Fig. 6A). Other mafl mutants were examined, with identical results (data not shown). These results show that disruption of $M A F 1$ significantly reduces the pathogenicity of $C$. lagenarium. However, when $20-\mu l$ drops of a conidial suspension of DMA5 were spotted on cucumber leaves, lesions formed more frequently than on spray-inoculated leaves, although the efficiency of lesion formation was still lower than that of the wild type (data not shown). Strains obtained from these lesions exhibited the original mafl phenotype in mycelial culture, appressorium formation, and pathogenicity, suggesting that the formation of lesions is not due to suppressor mutations (data not shown). These results indicate that MAF1 is important in the pathogenicity of $C$. lagenarium, but gene disruption of MAF1 did not cause complete loss of pathogenicity. To assess invasive growth ability, leaves were inoculated with mafl mutants via wounds (Fig. 6B). The melanin-deficient mutant 79215 , carrying a mutation in $P K S 1$, was used as a positive control (Takano et al., 1995). This strain is not pathogenic on intact leaves, because of a lack of appressorial melanization, but it is invasive at wound sites (Takano et al., 2000). Strain SGC1, isolated as a suppressor mutant from the PKA regulatory subunit (Rpk1) mutant, was used as a negative control. This strain does not cause lesions, even on leaves inoculated at wound sites (Takano et al., 2001a). Yellowish lesions formed on cucumber leaves at all wound sites spotted with a conidial suspension of DMA5 or the pks1 mutant 79215, but not on leaves spotted with SGC1. This result indicates that the mafl $\Delta$ strains retain the ability to grow invasively in host plant tissues. Thus, we conclude that the reduced pathogenicity of the mafl mutants is due to attenuated penetration of the host plant, probably caused by a failure of appressorium formation.

\section{$M A F 1$ is required in the early phase of appressorium formation, whereas $C M K 1$ is involved in appressorium maturation.}

The wild-type strain 104-T of C. lagenarium forms melanized appressoria on a glass surface at $24^{\circ} \mathrm{C}$ (Fig. 7A). How- ever, conidia of the wild-type strain incubated under certain conditions exhibited a phenotype morphologically similar to that of the mafl mutants. Conidia of the wild type incubated on PDAS nutrient agar (3.9\% PDA and 0.6 M sucrose) instead of glass germinated and produced elongated germ tubes without appressoria (Fig. 7D); $66 \%$ of germinating conidia of the wild type $(n=200)$ on PDAS had two rounds of nuclear division after $12 \mathrm{~h}$ of incubation, resulting in four nuclei, which seemed similar to the phenotype of the mafl mutants on an inductive glass surface. These similarities suggest a possibility that the nondifferentiation of appressoria of wild-type $C$. lagenarium on the nutrient agar is associated with the Maf1 MAPK cascade.

It has been shown by Takano et al. (2000) that the CMK1 MAPK gene is required for appressorium formation. We compared the morphological phenotypes of $\mathrm{cmkl}$ and mafl mutants during conidial incubation on an inductive glass surface. In previous studies, $c m k l$ mutants suspended in water failed to germinate on a glass surface, but the addition of yeast extract restored their germination (Takano et al., 2000). However, even when germination of the cmkl mutants was restored by yeast extract, the germinating conidia failed to form normal appressoria; rather, they formed swollen, appressorium-like structures that exhibited curling (Takano et al., 2000) (Fig. 7C). In the presence of yeast extract, the mafl mutants produced elongated germ tubes without forming any curled, swollen structures, the same as the mutants in water (Fig. 7B). This indicates that the morphological phenotype of the mafl mutants differs from that of the $c m k l$ mutants when they are incubated on an inductive glass surface, and it suggests that $M A F 1$ is required for the ear-

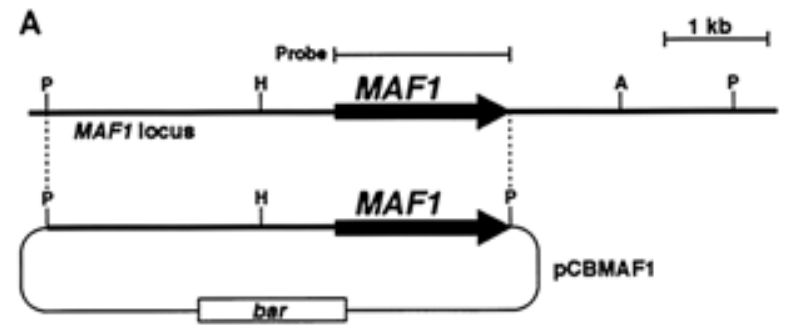

B

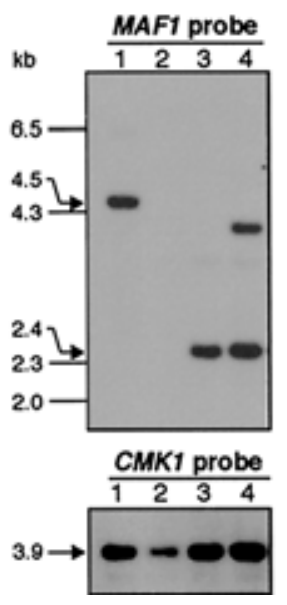

Fig. 3. Reintroduction of $M A F 1$ into the maf1 $\triangle$ strain. A, Plasmid pCBMAF1, carrying a bialaphos resistance gene and the 4.5-kb Pst I fragment containing $M A F 1$, was introduced into maf1 $\triangle$ strain DMA5, and bialaphos-resistant transformants were obtained. B, Genomic DNA gel blot analysis of MAF1-reintroduced transformants of DMA5. Genomic DNAs were extracted from the wild-type strain 104-T (lane 1), DMA5 (lane 2), and two MAF1-reintroduced transformants, RMA1 and RMA2 (lanes 3 and 4). All genomic DNA samples were digested by HindIII and Pst I. The blot was probed with the 1.7-kb MAF1 fragment shown in A. As a control, the blot was also probed with a fragment containing the $C M K 1$ gene (Takano et al., 2000). 
lier differentiation step of appressorium formation, whereas $C M K 1$ is involved in later maturation steps. The phenotype of mafl mutants on PDAS was similar to that of the mutants on an inductive glass surface (Fig. 7E). The cmkl mutants incubated on PDAS, like the wild type and the mafl mutants, also produced elongated germ tubes without forming curled swollen structures (Fig. 7F).

\section{DISCUSSION}

The mafl mutants, generated by targeting gene disruption, had growth rates similar to that of the wild type on nutrient media. This suggests that, like the Cmk1 MAPK, the Maf1 MAPK is dispensable for vegetative growth. However, the two MAPKs have different roles in fungal invasive growth in cucumber leaves. The mafl mutants formed lesions after inoculation at wound sites, indicating that $M A F 1$ is dispensable for invasive growth in the host plant, whereas $C M K 1$ is necessary for invasive growth (Takano et al., 2000). Similarly, the MPS1 MAPK gene of $M$. grisea, homologous to $M A F 1$, is not essential for invasive growth in rice (Xu et al., 1998). Also, conidiation was reduced in the mafl mutants, as it is in $M$. grisea mps 1 mutants, suggesting a conserved role of MPK1-type MAPK genes for conidiation in both $C$. lagenarium and $M$. grisea. We have shown that disruption of $C M K 1$ caused a reduction in conidiation (Takano et al., 2000), suggesting the involvement of two different MAPKs (Maf1 and Cmk1) in the conidiation of $C$. lagenarium.

The Mpk1-type MAPKs have been shown to be involved in the maintenance of cell wall integrity in several fungi, such as S. cerevisiae, Schizosaccharomyces pombe, Candida albicans, and M. grisea (Lee et al., 1993; Navarro-García et al., 1995; Toda et al., 1996; Xu et al., 1998). In contrast, we did not find any differences in sensitivity to cell-wall-digesting enzymes in the wild-type strain and mafl mutants of $C$. lagenarium, sug-

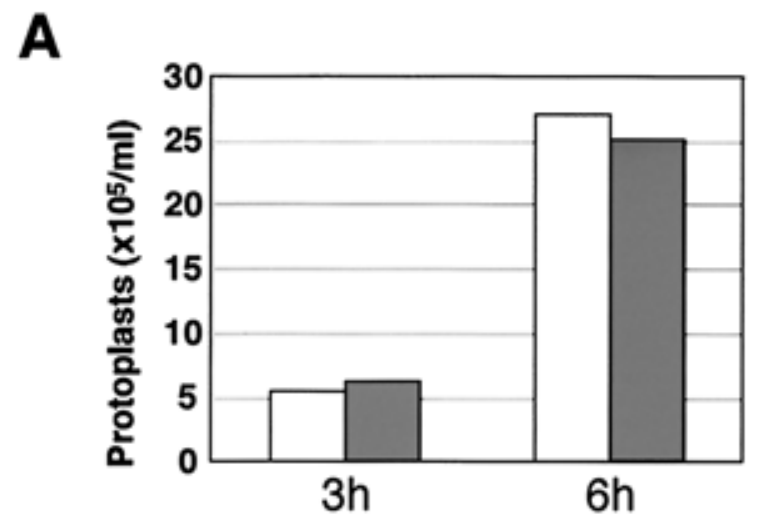

B
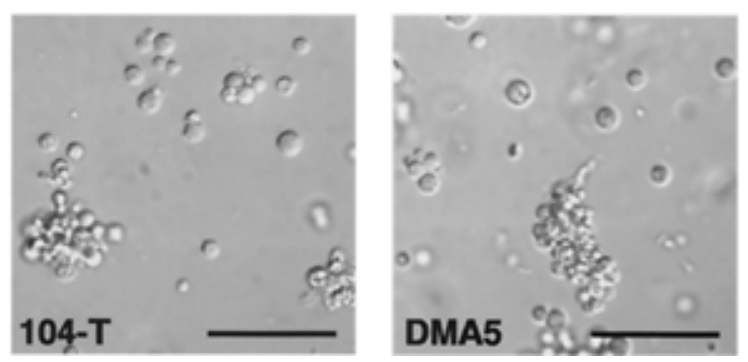

Fig. 4. Sensitivity of mafl mutants to cell-wall-digesting enzymes. A, Quantitative analysis of protoplasts released by mycelia of the wild-type strain 104-T (open bars) and mafl $\Delta$ strain DMA5 (shaded bars) 3 and $6 \mathrm{~h}$ after treatment with the cell-wall-digesting enzymes $\beta$-D-glucanase and Driselase. B, Protoplasts released from mycelia of 104-T and DMA5 $6 \mathrm{~h}$ after treatment with cell-wall-digesting enzymes. Bars $=50 \mu \mathrm{m}$. gesting that the MAF1-encoded MAPK is dispensable for cell wall integrity. There is a possibility that (1) Maf1 redundantly regulates the maintenance of cell wall integrity with one or more other unidentified signaling pathways or (2) Maf1 is not involved in the maintenance of cell wall integrity.

The FUS3/KSS1-type MAPK genes are commonly required for appressorium formation in $M$. grisea, C. heterostrophus, C. lagenarium, and P. teres (Lev et al., 1999; RuizRoldan et al., 2001; Takano et al., 2000; Xu and Hamer, 1996). In contrast with conserved roles of Fus3/Kss 1 MAPKs for appressorium formation, the different phenotypes in appressorium formation by $C$. lagenarium mafl and M. grisea mps 1 mutants imply that MPK1-type MAPK genes have diverse roles in appressorium formation in phytopathogenic fungi. In $S$. cerevisiae, the Mpk1 MAPK is involved in other aspects, including actin organization, in addition to the maintenance of cell wall integrity (Costigan and Snyder, 1994; Heinisch et al., 1999; Helliwell et al., 1998; Madden et al., 1997; Philips and Herskowitz, 1997). Our experiments using actin-depolymerizing drugs suggest that rearrangement of the actin cytoskeleton plays an important role in appressorium formation by C. lagenarium (E. Oshiro, Y. Takano, and T. Okuno, unpublished results). The inability of the mafl mutants to form appressoria might be due to a failure of the actin organization required for appressorium formation.

Few lesions formed on host plants spray-inoculated with the mafl mutants, but lesions formed more frequently on drop-inoculated leaves. These results indicate that the mafl mutants retained some pathogenicity, although they never formed appressoria. In Colletotrichum species, mechanical penetration using appressoria is thought to be a major pathogenic factor (Bechinger et al., 1999). The finding that the mafl mutants did not completely lose pathogenicity suggests that other pathogenicity factors are also involved in infection by $C$. lagenarium. In the drop inoculation tests, a big group of the mafl mutant conidia in conidial suspension drops may produce degrading enzymes or toxic compounds sufficient for penetration and lesion formation without appressoria.

M. grisea forms appressoria efficiently on hydrophobic surfaces but not on hydrophilic surfaces (Lee and Dean, 1994). In contrast, $C$. lagenarium forms appressoria on both hydrophobic and hydrophilic surfaces, although these surface properties do seem to affect germination efficiency (Takano et al., 2000). Also, M. grisea on a nutrient agar has been reported to produce elongated germ tubes without forming appressoria. This is similar to the phenotype of $C$. lagenarium on the nutrient agar (Adachi and Hamer, 1998; DeZwaan et al., 1999). These results imply that fungal pathogens commonly do not develop appressoria on nutrient agar via sensing mechanisms of environmental conditions. We showed that the mafl mutants on inductive surfaces exhibited a phenotype similar to that of the wild-type $C$. lagenarium on the nutrient agar, which suggests that incubation on the nutrient agar represses the appressorium differentiation pathway mediated by $M A F 1$. We consider that the Maf1 MAPK cascade is under putative environmental sensing mechanisms that control appressorium differentiation.

Comparison of the mafl mutants with the cmkl mutants on inductive glass surfaces suggests that $M A F 1$ is required for the early phase of appressorium formation, whereas $C M K 1$ is involved in appressorium maturation. The $c m k l$ mutants formed swollen, appressorium-like structures on a glass surface but not on the nutrient agar, indicating that they can respond to the glass surface signal for appressorium formation. These results suggest that $C M K 1$ is not involved in early appressorium differentiation mediated by contact on the inductive glass surface and that $C M K 1$ may function downstream of the contact-mediated pathway that triggers appressorium differentiation. 
In this study, we present evidence that two MAPKs, Maf1 and Cmk1, independently control different steps in appressorium development in C. lagenarium. Further studies to identify upstream regulators and downstream targets of these MAPK cascades will elucidate MAPK-mediated infection mechanisms of fungal pathogens and will offer novel strategies for protecting plants from fungal infection.

\section{MATERIALS AND METHODS}

\section{Fungal strains and culture conditions.}

C. lagenarium strain 104-T (stock culture of the Laboratory of Plant Pathology, Kyoto University) was used as the wildtype strain. All $C$. lagenarium cultures were maintained on PDA medium (3.9\% PDA, wt/vol) (Difco, Detroit, MI, U.S.A.) at $24^{\circ} \mathrm{C}$. Conidia were obtained by gently scraping cultures incubated for 7 days.

\section{MAF 1 cloning.}

A nested PCR was performed to isolate MAPK genes using cDNA synthesized from polyadenylated RNA prepared from conidia $2 \mathrm{~h}$ after the start of incubation for appressorium formation. Three degenerate primers-primer LVN, 5'-YTNGTNAAYGCNGAYTG-3' (LVNADC); primer CDF, 5'-GGACGGATCCTG-
YGAYTTYGGNYTNGC-3' (CDFGLA); and primer WYR, 5'CCCAGAATTCTCNGGNGCNCKRTACCA-3' (WYRAPE)were designed, based on the conserved amino acids in Mpk1type MAPKs in other organisms. Primer CDF contains a terminal BamHI site. Primer WYR contains a terminal EcoRI site. The first PCR was performed with primers LVN and WYR with Ex Taq polymerase (Takara, Ohtsu, Japan). Nested PCR was performed with primers $\mathrm{CDF}$ and WYR. PCR conditions were 30 cycles at $94^{\circ} \mathrm{C}$ for $1 \mathrm{~min}, 52^{\circ} \mathrm{C}$ for $2 \mathrm{~min}$, and $72^{\circ} \mathrm{C}$ for $2 \mathrm{~min}$, with a final extension at $72^{\circ} \mathrm{C}$ for $5 \mathrm{~min}$. Nested PCR products were digested with BamHI and EcoRI, cloned into pBS $\left(\mathrm{KS}^{-}\right)$ (Stratagene, La Jolla, CA, U.S.A.), and sequenced. Clone pMP1, having homology with $S$. cerevisiae MPK1 and M. grisea MPS1, was used as a probe to screen genomic clones containing the $M A F 1$ gene from the $C$. lagenarium cosmid library. The DNA sequence was determined with a Big Dye terminator cycle sequencing ready reaction kit (Applied Biosystems, Warrington, U.K.) and an automated DNA sequencer (Applied Biosystems Model 310).

\section{Fungal transformation.}

Preparation of protoplasts and transformation of $C$. lagenarium were performed according to a method described previously (Takano et al., 2001b). Hygromycin- and bialaphos-resistant
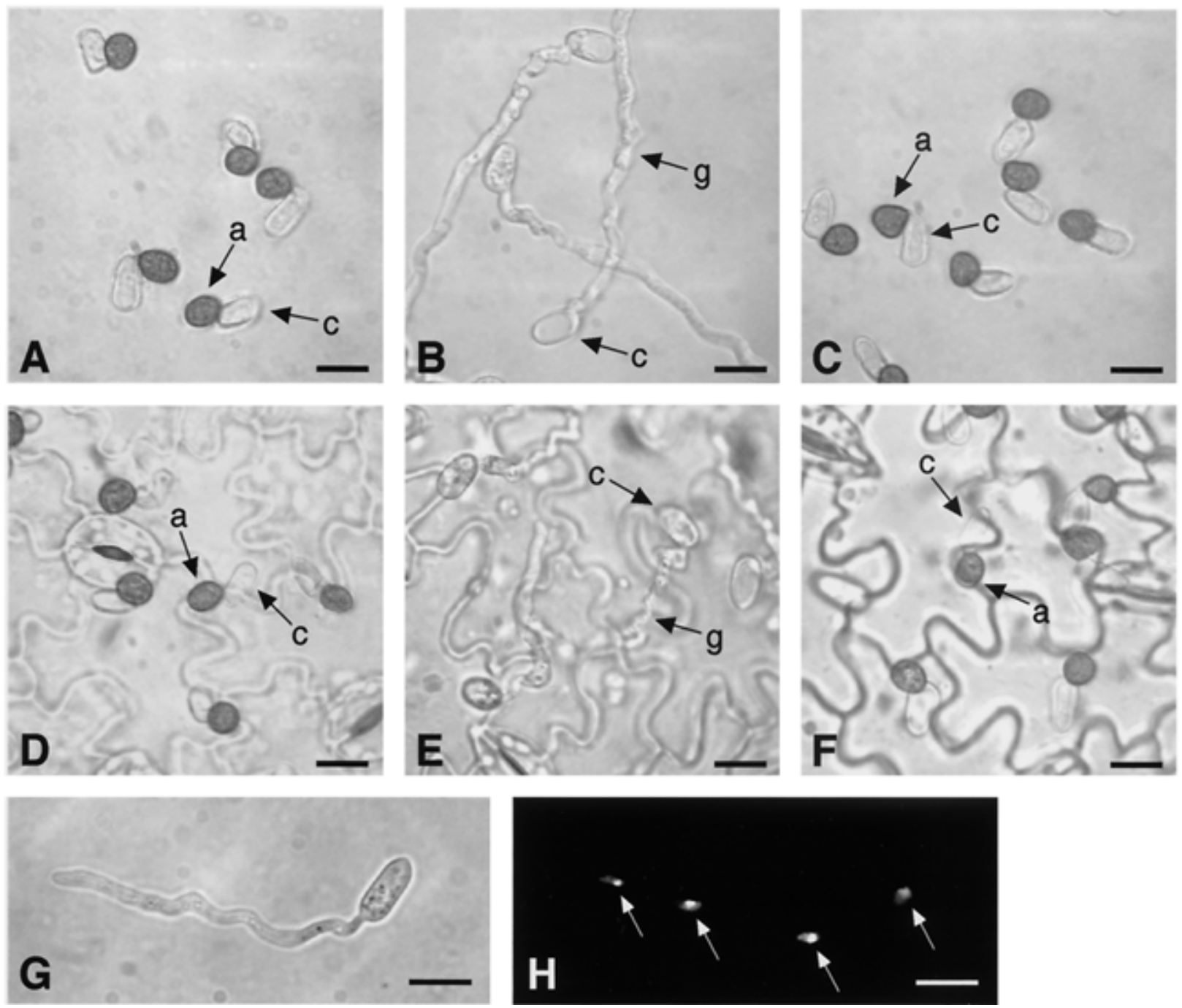

Fig. 5. MAF1 is essential for appressorium formation. A-F, Conidia of the wild-type strain 104-T (A and D), the maf1 mutant DMA5 (B and E), and the MAF1-reintroduced transformant RMA1 (C and F) incubated for $12 \mathrm{~h}$ on glass slides $(\mathbf{A}-\mathbf{C})$ or on the lower epidermis of cucumber cotyledons $(\mathbf{D}-\mathbf{F})$ : a, appressorium; c, conidium; g, germ tube. $\mathbf{G}$ and $\mathbf{H}$, Conidia of DMA5 incubated on glass for $12 \mathrm{~h}$ and then fixed and stained with 4',6-diamidino-2phenylindole dihydrochloride (DAPI) to visualize nuclei (arrows): differential interference contrast image (G) and DAPI image $(\mathbf{H})$. Bars $=10 \mu \mathrm{m}$. 
A

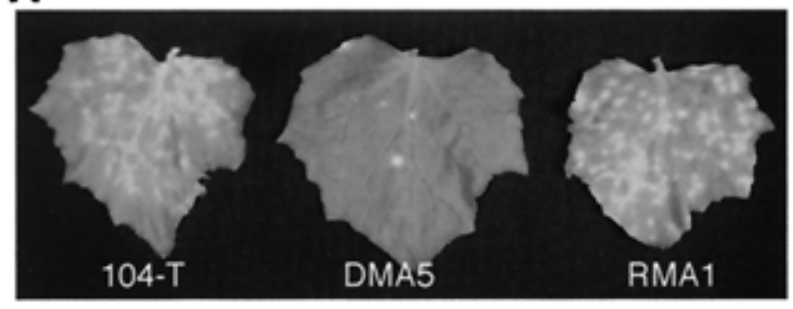

B

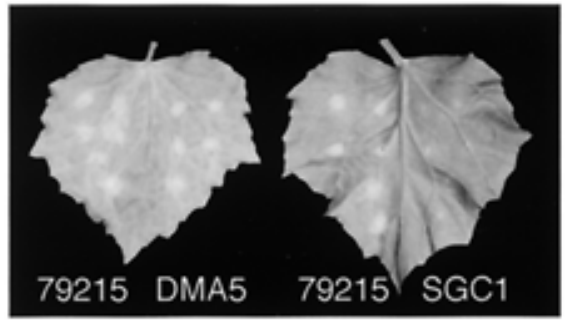

Fig. 6. $M A F 1$ is required for fungal pathogenicity. A, Detached cucumber leaves sprayed with suspensions of conidia of the wild-type strain 104-T, mafl $\triangle$ mutant DMA5, and the MAF1-reintroduced transformant RMA1 and incubated at $25^{\circ} \mathrm{C}$ for 7 days. B, Inoculation assay of wounded cucumber leaves. Detached cucumber leaves wounded by scratching with a needle and inoculated by spotting wound sites with suspensions of conidia of DMA5 and SGC1, a growth suppressor mutant isolated from the PKA regulatory subunit mutant (Takano et al., 2001a), on the right half of the leaf, and with the melanin-deficient mutant 79215 ( $p k s 1$ ) on the left half of the leaf as a positive control. Inoculated leaves were incubated at $25^{\circ} \mathrm{C}$ for 7 days. transformants were selected on regeneration medium containing hygromycin B $(100 \mu \mathrm{g} / \mathrm{ml})$ (Wako Pure Chemicals, Osaka, Japan) and bialaphos $(250 \mu \mathrm{g} / \mathrm{ml})$ (kindly provided by Nobuyuki Fuchigami, Meiji Seika Kaisha), respectively.

\section{Genomic DNA blot analysis.}

Total DNA of C. lagenarium was isolated from mycelia by a method described previously (Takano et al., 1997). DNA digestion, gel electrophoresis, labeling of probes, and hybridization were performed according to the manufacturers' instructions and standard methods (Sambrook et al., 1989). DNA probes were labeled digoxygenin-dUTP (Roche Molecular Biochemicals) with the BcaBEST digoxygenin labeling kit (Takara). Hybridization was carried out as described previously (Takano et al., 1997).

\section{Plasmid construction.}

To construct the gene disruption vector pGDMAF1, two DNA fragments (a 7.5-kb ApaI fragment and a 6.7-kb Pst I fragment) containing the $M A F 1$ gene were subcloned into $\mathrm{pBSII}\left(\mathrm{KS}^{-}\right)$(Stratagene) from the cosmid clone, to produce pMAFA and pMAFP, respectively. The 1.1-kb fragment containing the $3^{\prime}$ flanking region of $M A F 1$ was amplified by PCR using primer MAFD1A (5'-CGCAAGGGGCCCAGGTTATGACGAGAGCG-3') and M13 reverse primer from pMAFA. Primer MAFD1A contains a terminal ApaI site. PCR was performed with LA-taq polymerase (Takara) for high fidelity of DNA synthesis. The amplified fragment was digested with ApaI and introduced into ApaI sites of pCB1636 (Sweigard et al., 1997), containing the $h p h$ gene, to produce plasmid pCBMAF3A. The 2.8-kb fragment containing the 5' flanking region of $M A F 1$ was amplified by PCR using primer MAFD2P (5'-ACAAAACTGCAGTGCTTCTTGTTGAATCT-3') and
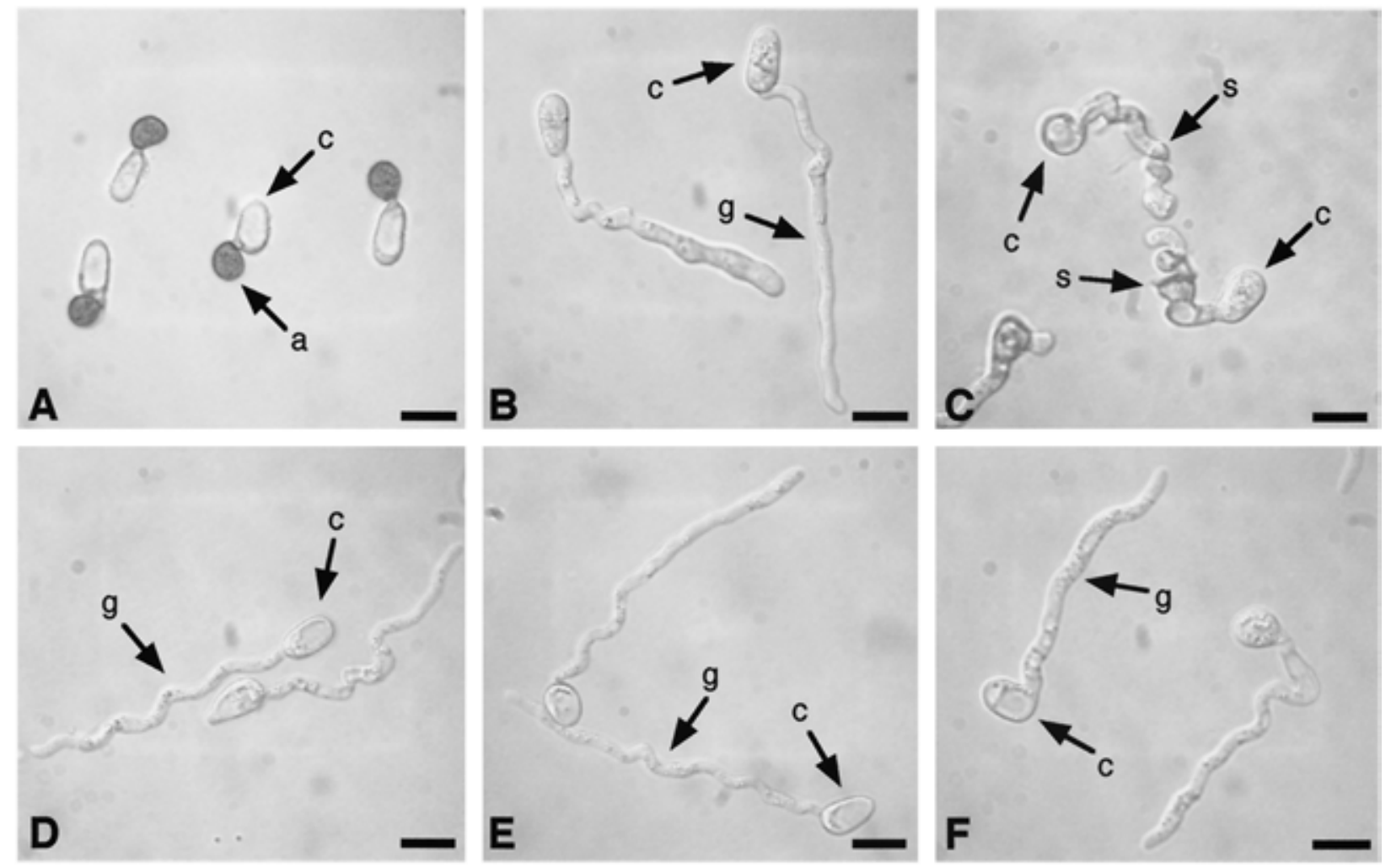

Fig. 7. Morphological phenotype of the wild-type strain 104-T (A and D), the mafl mutant DMA5 (B and E), and the cmk1 mutant DCM1 (C and F) on glass slides (A-C) and on nutrient agar (D-F): a, appressorium; c, conidium; g, germ tube; s, swollen appressorium-like structure. Conidia of the wildtype strain and the mafl mutant suspended in $0.1 \%$ yeast extract were incubated for $12 \mathrm{~h}$ at $24^{\circ} \mathrm{C}$. Conidia of the $\mathrm{cmk} 1$ mutant in $0.1 \%$ yeast extract were incubated for $18 \mathrm{~h}$ at $24^{\circ} \mathrm{C}$, because of delayed germination of this mutant (Takano et al., 2000). Bars $=10 \mu \mathrm{m}$. 
M13 forward primer from pMAFP. Primer MAFD2P contains a terminal $P s t$ I site. The amplified fragment was digested with Pst I and introduced into Pst I sites of pCBMAF3A, to produce plasmid pGDMAF1. To construct plasmid pCBMAF1 for use in a complementation test of the mafl mutant, a genomic fragment was amplified from pMAFP by PCR using primer MAF1CP (5'-AAACTGCAGTGTCGTGTGTTCATTCG-3') and M13 forward primer. Primer MAF1CP contains a terminal PstI site. The amplified fragment was digested with Pst I and introduced into Pst I sites of pCB1531, containing a bialaphosresistant gene (Sweigard et al., 1997) to produce pCBMAF1.

\section{Pathogenicity tests.}

Conidia were collected from 7-day-old PDA cultures and resuspended in water (approximately $5 \times 10^{5}$ conidia per $\mathrm{ml}$ ). The conidial suspension was sprayed on the surface of detached cucumber leaves (Cucumis sativus L. 'Suyo'). Inoculated leaves were placed in humid petri dishes and incubated at $25^{\circ} \mathrm{C}$ for 7 days. For microscopic observation, $20 \mu \mathrm{l}$ of conidial suspension was spotted on the lower epidermis of cucumber cotyledons. The epidermal layers were peeled off and observed under light microscopy $12 \mathrm{~h}$ after inoculation (Takano et al., 1997). For leaf wound inoculation, wounds (1 to $\left.2 \mathrm{~mm}^{2}\right)$ were produced by scratching with $26 \mathrm{G}_{1 / 2}$ needles, and leaves were inoculated with a conidial suspension spotted on the wound sites.

\section{Microscopy.}

For synchronous infection-related morphogenesis on glass slides, $20 \mu \mathrm{l}$ of a conidial suspension $\left(1 \times 10^{5}\right.$ conidia per $\mathrm{ml}$ in $0.1 \%$ yeast extract solution) was placed on an eight-well multitest glass slide (INC Biochemicals, Aurora, OH, U.S.A.) and incubated at $24^{\circ} \mathrm{C}$ (Takano et al., 2001b). After $1 \mathrm{~h}$ of incubation, $0.1 \%$ yeast extract solution was changed to distilled water, and the samples were subjected to further incubation. For incubation on PDAS medium, containing PDA (3.9\%, $\mathrm{wt} / \mathrm{vol}$ ) and $0.6 \mathrm{M}$ sucrose (Difco, Detroit, MI, U.S.A.), the glass slide was coated with PDAS prior to placement of the conidial suspension. In morphological comparisons of the mafl mutant with the cmkl mutant, conidia of the tested strains were incubated continuously in the presence of yeast extract to restore germination of the $\mathrm{cmkl}$ mutant. For staining of nuclei, samples on glass slides were fixed with $3.75 \%$ formaldehyde, $50 \mathrm{mM}$ phosphate buffer ( $\mathrm{pH} 7.0$ ), and $0.2 \%$ Triton $\mathrm{X}-100$ at room temperature for $30 \mathrm{~min}$ (Takano et al., 2001b). Fixed samples were rinsed with distilled water, stained with 4',6-diamidino-2-phenylindole dihydrochloride (DAPI, $1 \mu \mathrm{g} / \mathrm{ml}$ ) (Sigma, St. Louis, MO, U.S.A.) for $30 \mathrm{~min}$, and then rinsed again with distilled water. Stained cells were observed with a microscope with a Zeiss filter set (excitation $365 \mathrm{~nm}$, dichroic $395 \mathrm{~nm}$, emission $420 \mathrm{~nm}$ ). Images were captured with a chilled charge-coupled device camera (Argus 50, Hamamatsu Photonics, Hamamatsu, Japan).

For cell wall integrity assays, mycelia of tested strains grown in PSY (potato-sucrose broth supplemented with $0.2 \%$ yeast extract) were transferred to a solution of $1.2 \mathrm{M} \mathrm{MgSO}_{4}$ and $10 \mathrm{mM} \mathrm{Na} \mathrm{HPO}_{4}$ with $\beta$-D-glucanase $(5 \mathrm{mg} / \mathrm{ml}$ ) (InterSpex Products, Inc., San Mateo, CA, U.S.A.) and Driselase (10 $\mathrm{mg} / \mathrm{ml}$ ) (Sigma) and incubated at $30^{\circ} \mathrm{C}$. Protoplasts released from mycelia 3 and $6 \mathrm{~h}$ after the enzyme treatment were examined under a microscope, and the concentration of protoplasts in a sample solution was calculated.

\section{ACKNOWLEDGMENTS}

We thank Kazuyuki Mise and Iwao Furusawa for valuable suggestions and support during the course of this work. We are grateful to Nobuyuki
Fuchigami for providing bialaphos. This work was supported in part by a Grant-in-Aid for Scientific Research on priority area (A) "Molecular Mechanisms of Plant-Microbe Interaction Toward Production of Disease Resistant Plants" and by a Grant-in-Aid for Scientific Research (A) (13306005) from the Ministry of Education, Culture, Sports, Science and Technology, Japan.

\section{LITERATURE CITED}

Adachi, K., and Hamer, J. E. 1998. Divergent cAMP signaling pathways regulate growth and pathogenesis in the rice blast fungus Magnaporthe grisea. Plant Cell 10:1361-1373.

Agrios, G. N. 1988. Plant Pathology. 3rd ed. Academic Press, San Diego, CA.

Bechinger, C., Giebel, K. F., Schnell, M., Leiderer, P., Deising, H. B., and Bastmeyer, M. 1999. Optical measurements of invasive forces exerted by appressoria of a plant pathogenic fungus. Science 285:1896-1899.

Choi, W., and Dean, R. A. 1997. The adenylate cyclase gene MAC1 of Magnaporthe grisea controls appressorium formation and other aspects of growth and development. Plant Cell 9:1973-1983.

Costigan, C., and Snyder, M. 1994. SLK1, a yeast homolog of MAP kinase activators, has a RAS/cAMP-independent role in nutrient sensing. Mol. Gen. Genet. 243:286-296.

DeZwaan, T. M., Carroll, A. M., Valent, B., and Sweigard, J. A. 1999. Magnaporthe grisea Pth11p is a novel plasma membrane protein that mediates appressorium differentiation in response to inductive substrate cues. Plant Cell 11:2013-2030.

Di Pietro, A., Garcia-MacEira, F. I., Meglecz, E., and Roncero, M. I. 2001. A MAP kinase of the vascular wild fungus Fusarium oxysporum is essential for root penetration and pathogenesis. Mol. Microbiol. 39:11401152.

Heinisch, J. J., Lorberg, A., Schmitz, H.-P., and Jacoby, J. J. 1999. The protein kinase $\mathrm{C}$-mediated MAP kinase pathway involved in the maintenance of cellular integrity in Saccharomyces cerevisiae. Mol. Microbiol. 32:671-680.

Helliwell, S. P., Schmidt, A., Ohya, Y., and Hall, M. N. 1998. The Rho1 effector, but not Bni1, mediates signaling from Tor2 to the actin cytoskeleton. Curr. Biol. 8:1211-1214.

Herskowitz, I. 1995. MAP kinase pathways in yeast: For mating and more. Cell 80:187-197.

Hoch, H. C., Staples, R. C., Whitehead, B., Comeau, J., and Wolfe, E. D. 1987. Signaling for growth orientation and cell differentiation by surface topography in Uromyces. Science 234:1659-1662.

Hunter, T., and Plowman, G. D. 1997. The protein kinases of budding yeast: Six score and more. Trends Biochem. Sci. 22:18-22

Inagaki, A., Takano, Y., Kubo, Y., Mise, M., and Furusawa, I. 2000. Construction of an equalized cDNA library from Colletotrichum lagenarium and its application to the isolation of differentially expressed genes. Can. J. Microbiol. 46:1-9.

Lee, K. S., Irie, K., Gotoh, Y., Watanabe, Y., Araki, H., Nishida, E., Matsumoto, K., and Levin, D. E. 1993. A yeast mitogen-activated protein kinase homolog (Mpk1p) mediates signaling by protein kinase C. Mol. Cell Biol. 13:3067-3075.

Lee, Y.-H., and Dean, R. A. 1993. cAMP regulates infection structure formation in the plant pathogenic fungus Magnaporthe grisea. Plant Cell 5:693-700.

Lee, Y.-H., and Dean, R. A. 1994. Hydrophobicity of contact surface induces appressorium formation in Magnaporthe grisea. FEMS Microbiol. Lett. 115:71-76.

Lev, S., Sharon, A., Hadar, R., Ma, H., and Horwitz, B. A. 1999. A mitogenactivated protein kinase of the corn leaf pathogen Cochliobolus heterostrophus is involved in conidiation, appressorium formation, and pathogenicity: Diverse roles for mitogen-activated protein kinase homologues in foliar pathogens. Proc. Natl. Acad. Sci. USA 96:1354213547.

Madden, K., Sheu, Y. J., Baetz, K., Andrews, B., and Snyder, M. 1997. SBF cell cycle regulator as a target of the yeast PKC-MAP kinase pathway. Science 275:1781-1784.

Mayorga, M. E., and Gold, S. E. 1999. A MAP kinase encoded by the $u b c 3$ gene of Ustilago maydis is required for filamentous growth and full virulence. Mol. Microbiol. 34:485-497.

Muller, P., Aichinger, C., Feldbrugge, M., and Kahmann, R. 1999. The MAP kinase Kpp2 regulates mating and pathogenic development in Ustilago maydis. Mol. Microbiol. 34:1007-1017.

Navarro-García, F., Sanchez, M., Pla, J., and Nombela, C. 1995. Functional characterization of the $M K C 1$ gene of Candida albicans, which encodes a mitogen-activated protein kinase homolog related to cell integrity. Mol. Cell Biol. 15:2197-2206.

Nishida, E., and Gotoh, Y. 1993. The MAP kinase cascade is essential for diverse signal transduction pathways. Trends Biochem. Sci. 18:128-131. 
Philips, J., and Herskowitz, I. 1997. Osmotic balance regulates cell fusion during mating in Saccharomyces cerevisiae. J. Cell Biol. 138:961-974.

Ruiz-Roldan, M. C., Maier, F. J., and Schafer, W. 2001. PTK1, a mitogenactivated-protein kinase gene, is required for conidiation, appressorium formation, and pathogenicity of Pyrenophora teres on barley. Mol. Plant-Microbe Interact. 14:116-125.

Sambrook, J., Fritsch, E. F., and Maniatis, T. 1989. Molecular Cloning: A Laboratory Manual. 2nd ed. Cold Spring Harbor Laboratory, Cold Spring Harbor, NY.

Schaeffer, H. J., and Webber, M. J. 1999. Mitogen-activated protein kinases: Specific messages from ubiquitous messengers. Mol. Cell Biol. 19:2435-2444.

Sweigard, J., Chumley, F., Carroll, A., Farrall, L., and Valent, B. 1997. A series of vectors for fungal transformation. Fungal Genet. Newsl. 44:52-55.

Takano, Y., Kubo, Y., Shimizu, K., Mise, K., Okuno, T., and Furusawa, I 1995. Structural analysis of $P K S 1$, a polyketide synthase gene involved in melanin biosynthesis in Colletotrichum lagenarium. Mol. Gen. Genet. 249:162-167.

Takano, Y., Kubo, Y., Kawamura, C., Tsuge, T., and Furusawa, I. 1997. The Alternaria alternata melanin biosynthesis gene restores appressorial melanization and penetration of cellulose membranes in the melanin-deficient albino mutant of Colletotrichum lagenarium. Fungal Genet. Biol. 21:131-140.

Takano, Y., Kikuchi, T., Kubo, Y., Hamer, J. E., Mise, K., and Furusawa, I. 2000. The Colletotrichum lagenarium MAP kinase gene $C M K 1$ regulates diverse aspects of fungal pathogenesis. Mol. Plant-Microbe Interact. 13:374-383.
Takano, Y., Komeda, K., Kojima, K., and Okuno, T. 2001a. Proper regulation of cyclic AMP-dependent protein kinase is required for growth, conidiation, and appressorium function in the anthracnose fungus $\mathrm{Col}$ letotrichum lagenarium. Mol. Plant-Microbe Interact. 14:1149-1157.

Takano, Y., Oshiro, E., and Okuno, T. 2001b. Microtubule dynamics during infection-related morphogenesis of Colletotrichum lagenarium. Fungal Genet. Biol. 34:107-121.

Thompson, J. D., Higgins, D. G., and Gibson, T. J. 1994. CLUSTALW: Improving the sensitivity of progressive multiple sequence alignment through sequence weighting, position-specific gap penalties and weight matrix choice. Nucleic Acids Res. 22:4673-4680.

Toda, T., Dhut, S., Superti-furga, G., Gotoh, Y., Nishida, E., Sugiura, R., and Kuno, K. 1996. The fission yeast $p m k 1+$ gene encodes a novel mitogen-activated protein kinase homolog which regulate cell integrity and functions coordinately with the protein kinase $\mathrm{C}$ pathway. Mol Cell Biol. 16:6752-6764.

$\mathrm{Xu}$, J.-R. 2000. MAP kinases in fungal pathogens. Fungal Genet. Biol 31:137-152

Xu, J.-R., and Hamer, J. E. 1996. MAP kinase and cAMP signaling regulate infection structure formation and pathogenic growth in the rice blast fungus Magnaporthe grisea. Genes Dev. 10:2696-2706.

Xu, J.-R., Staiger, C. J., and Hamer, J. E. 1998. Inactivation of the mitogenactivated protein kinase Mps1 from the rice blast fungus prevents penetration of host cells but allows activation of plant defense responses. Proc. Natl. Acad. Sci. USA 95:12713-12718.

Zheng, L., Campbell, M., Murray, J., Lam, S., and Xu, J.-R. 2000. The $B M P 1$ gene is essential for pathogenicity in the gray mold fungus Botrytis cinerea. Mol. Plant-Microbe Interact. 13:724-732. 